\title{
Thermodynamic measure of the magnetoelectric coupling in a three-dimensional topological insulator
}

\author{
Doron L. Bergman ${ }^{1}$ and Gil Refael ${ }^{1,2}$ \\ ${ }^{1}$ Physics Department, California Institute of Technology, MC 114-36, 1200 East California Boulevard, Pasadena, California 91125, USA \\ ${ }^{2}$ Dahlem Center for Complex Quantum Systems and Fachbereich Physik, Freie Universität Berlin, 14195 Berlin, Germany
}

(Received 20 July 2012; published 8 January 2013)

\begin{abstract}
We show that the magnetoelectric coupling in three-dimensional (strong) topological insulators is related to a second derivative of the bulk magnetization. The formula we derive is the nonlinear-response analog of the Streda formula for Hall conductivity [Streda, J. Phys. C 15, L717 (1982)], which relates the Hall conductivity to the derivative of the magnetization with respect to the chemical potential. Our finding allows one to extract the magnetoelectric coefficient by measuring the magnetization, while varying the chemical potential and one more perturbing field. The relation we find also makes transparent the effect of disorder on the magnetoelectric response, which occurs only through the density of states, and has no effect when the system is gapped.
\end{abstract}

DOI: 10.1103/PhysRevB.87.024202

PACS number(s): 72.10.-d, 71.20.-b, 72.20.-i

\section{INTRODUCTION}

The quantum Hall effect ${ }^{1}$ (QHE) was the first experimental instance where a transport coefficient (the Hall conductivity in $d=2$ ) was quantized. ${ }^{2}$ Finding an analogous system in $d=3$ remained an unrealized dream of the condensed matter community, for many years. Recently, however, this has changed. The discovery of the topological insulator, ${ }^{3-5}$ and specifically the three-dimensional (3D) strong topological insulator (STI), ${ }^{6,7}$ has finally realized the dream of a $d=3$ analog of the QHE. In the context of the QHE, Smrcka and Streda. ${ }^{8,9}$ proved an insightful relation between the Hall conductivity and a second derivative of a thermodynamic potential,

$$
\sigma_{\text {Hall }}=\frac{1}{2}\left(\sigma_{x y}-\sigma_{y x}\right)=-\left.\frac{\partial \rho}{\partial B}\right|_{\mu}=-\left.\frac{\partial M}{\partial \mu}\right|_{B},
$$

where $\rho$ is the charge density, $M$ the orbital magnetization per unit volume (perpendicular to the $2 \mathrm{D}$ system), $B$ the external magnetic field, and $\mu$ the chemical potential. Here and throughout the paper we use units where $c=e=\hbar=1$.

Motivated by the analogy between the $d=2 \mathrm{QHE}$ and the $d=3 \mathrm{STI}$, in this paper we show that the magnetoelectric coefficient characterizing the STI is also related to a thermodynamic derivative. We use an alternative formulation of the magnetoelectric effect, as a current response defined by

$$
\mathbf{J}=\frac{\chi}{2 \pi} \nabla \phi \times \mathbf{E},
$$

where $\phi$ is an external field odd under inversion and time reversal, and $\chi$ is the magnetoelectric response coefficient, and show that

$$
\chi=-\frac{2 \pi}{3} \frac{\partial^{2} \rho}{\partial B^{a} \partial\left(\partial_{a} \phi\right)}=-\frac{2 \pi}{3} \frac{\partial^{2} M_{a}}{\partial \mu \partial\left(\partial_{a} \phi\right)} .
$$

There are a number of merits to this result, similar to those of (1). First, (1) suggests that we can measure the Hall conductivity in the QHE by doing a thermodynamic measurement-by varying the chemical potential through a back gate and measuring the magnetization of the sample. Calculating the derivative of the magnetization with respect to the gate voltage should give $\sigma_{\text {Hall }}$. Similarly, with (3) we can measure $\chi$ by simultaneously varying the chemical potential $\mu$ and the gradient $\left(\partial_{a} \phi\right)$, while measuring the magnetization of the sample. Second, the effects of disorder on (1) and (3) are entirely included in the density of states $D(\epsilon)$ through the particle density $\rho=\int d \epsilon f(\epsilon) D(\epsilon)$, where $f(\epsilon)$ is the Fermi-Dirac distribution. Disorder will reduce the effective gap in the spectrum, compared with the clean limit, ${ }^{10}$ but otherwise will not change anything for an insulator, as long as the chemical potential remains in the gap.

The magnetoelectric effect ${ }^{11,12}$ is usually formulated as an anomalous term appearing in the action for the electromagnetic fields in an insulator $S_{E B}=\frac{1}{2 \pi} \int d \mathbf{r} d t P_{3} \mathbf{E} \cdot \mathbf{B}$, with $P_{3}$ the magnetoelectric coefficient. Under inversion, $\mathbf{E}$ is odd, and under time reversal $\mathbf{B}$ is odd, so $P_{3}$ is odd under both. The authors of Ref. 11 showed that $P_{3}$ takes on values modulo 1 , and so in a material with either time-reversal or inversion symmetry (or both), it can take on the values $P_{3}=0, \frac{1}{2}$. The value $P_{3}=\frac{1}{2}$ then characterizes the STI. This value can in principle be measured ${ }^{12}$ as

$$
P_{3} \delta_{a b}=2 \pi \frac{\partial M_{a}}{\partial E^{b}}=2 \pi \frac{\partial P_{a}}{\partial B^{b}},
$$

where the latin letters $a, b=x, y, z$ denote spatial directions. Here and throughout the paper we will use the Einstein summation convention.

At this point in time, a number of materials have been identified as topological insulators by using spectroscopy to characterize their unique surface states ${ }^{13-17}$ (an odd number of Dirac points). However, thus far measuring the magnetoelectric coefficient has proved challenging. The materials, by and large, have proven rather poor insulators, with significant carrier concentration, in some cases even a bulk Fermi surface appearing. ${ }^{18-24}$ In a bulk metal dc electric fields are screened, making it impossible to measure (4) directly.

Many other indirect ways have been proposed to detect the magnetoelectric effect, most of them relying on the surface states in the STI, ${ }^{11,12,25-35}$ and on the Witten effect. ${ }^{11,36,37}$ The magnetoelectric effect at the surface appears as a consequence of the material boundary, where $P_{3}$ has a sharp jump, and the Witten effect appears as a consequence of a flux monopole entering the STI. Our motivation in this work 
is to generalize the measurable effects of the magnetoelectric coupling, beyond the surface and Witten effects, to response in the bulk. This can be achieved, as we explain below, by using the field $\phi$ imitating the fourth vector potential component in $4 \mathrm{D}, A_{4}$, and replacing $P_{3}$. With this extra field $\phi$, we reformulate the magnetoelectric effect with (2), and find the thermodynamic relation (3) similar to (1). Formulated in this way, we will also be able to talk about magnetoelectric effects in gapless systems, as was recently explored in Refs. 38 and 39.

The paper is organized as follows. In Sec. II we present a rough derivation of our main result (a rigorous derivation is detailed in Appendix B). In Sec. III we discuss the implications of our result (3), followed by Sec. IV where we use a realistic model for the band structure of $\mathrm{Bi}_{2} \mathrm{Se}_{3}$ as an example for using the formalism we introduce here, and demonstrating the qualitative signatures of a topological insulator as opposed to a gapless system. We end with concluding remarks in Sec. V.

\section{DERIVATION OF THE THERMODYNAMIC RELATION}

Since the STI is the $d=3$ analog of the QHE, we look to $d=2$ for inspiration. First, the analog of magnetoelectric coupling in a gapless metal ${ }^{38,39}$ is the intrinsic anomalous Hall effect (AHE) ${ }^{40-43}$ in metals, where time-reversal symmetry is broken, a large clean-limit contribution to the Hall conductivity appears. The general expression for the intrinsic AHE contribution to the Hall conductivity becomes quantized once the chemical potential is in a gap of the band structure, giving the integer QHE. In a metal the effect of disorder cannot be neglected, and there are additional contributions to the Hall conductivity. ${ }^{43}$ The interplay of the intrinsic AHE and disorder can be understood using the so-called Bastin formula ${ }^{8,44,45}$ for the electric conductivity tensor, which distinguishes between contributions from states at the Fermi energy (the Fermi surface) $\sigma^{\mathrm{I}}$ and contributions from all filled states (the Fermi sea) $\sigma^{\text {II }}$. In an insulator, there are no bulk states at the Fermi energy, giving $\sigma^{\mathrm{I}}=0$. Smrcka and Streda ${ }^{8,9}$ were able to show that the $\sigma^{\mathrm{II}}$ contribution can be related to a derivative of the orbital magnetization,

$$
\sigma_{\text {Hall }}^{\text {II }}=-\left.\frac{\partial \rho(\mu)}{\partial B}\right|_{\mu}=-\left.\frac{\partial M}{\partial \mu}\right|_{B} .
$$

Here $M$ and $B$ are the orbital magnetization and magnetic field, respectively, ignoring Zeeman coupling to the electron spin. The formula holds in both gapped and gapless systems. For an insulator, $\sigma^{\mathrm{I}}=0$, and the total Hall conductivity is reduced to (1).

The result (1) can be anticipated from the following considerations. In an insulator, with no dissipative currents, the only currents possible are persistent currents related to the orbital magnetization $\mathbf{J}=\boldsymbol{\nabla} \times \mathbf{M}$. The electric field is found from $\mathbf{E}=-\nabla \mu(\mathbf{x})$. Assuming the magnetization is an entirely local function of the intensive thermodynamic quantities $M_{a}=M_{a}(T, \mathbf{B}, \mu(\mathbf{x}))$, we find

$$
J^{a}=\epsilon^{a b c} \partial_{b} M_{c}=\epsilon^{a b c} \frac{\partial M_{c}}{\partial \mu} \frac{\partial \mu}{\partial x^{b}}=-\epsilon^{a b c} \frac{\partial M_{c}}{\partial \mu} E_{b},
$$

resulting in $\sigma_{x y}=-\frac{\partial M_{z}}{\partial \mu}$. The second equality in (5) is due to a Maxwell relation. ${ }^{46,47}$
As mentioned earlier, (5) indicates that the $\sigma^{\mathrm{II}}$ contribution can be found by doing a thermodynamic measurement. However, the measured magnetization will include both the orbital and Zeeman contributions to the magnetization, while (5) involves the orbital magnetization alone. For an insulator one can argue that the magnetization due to Zeeman coupling does not vary with chemical potential, and therefore measuring the derivative of the total magnetization will give the same result as if we were measuring the orbital magnetization alone. For a metal, on the other hand, the Zeeman effect magnetization can depend on the chemical potential, for instance in Pauli paramagnetism. Therefore, measuring the total magnetization will give a quantitatively accurate measure of $\sigma^{\mathrm{II}}$ only in an insulator. Still, it will be useful in finding qualitative differences - it will exhibit quantization when the bulk is gapped. While such a measurement is conceptually straightforward, in practice it is more difficult than measuring the Hall effect through electric currents. However, it has been carried out. ${ }^{48,49}$ Next we will present the analog of (5) in the $d=3$ STI.

In order to deal with a possibly gapless spectrum, as well as with disorder, we will have to formulate the magnetoelectric coupling in a slightly different way from that in Refs. 11 and 12. The magnetoelectric effect relates the magnetization to an applied electric field $\mathbf{M}=\frac{P_{3}}{2 \pi} \mathbf{E}$ equivalent to (4). The Hall conductivity is well defined for both an insulator and a metal because it is defined through a current response to an external field, $J_{x}=\sigma_{x y} E_{y}$. For an insulator, $\mathbf{J}=\nabla \times \mathbf{M}$, and using Faraday's law $\boldsymbol{\nabla} \times \mathbf{E}=-\partial_{t} \mathbf{B}$, we get

$$
\mathbf{J}=\frac{1}{2 \pi} \nabla P_{3} \times \mathbf{E}-\frac{P_{3}}{2 \pi} \partial_{t} \mathbf{B} .
$$

The only dc (static) response comes from the first term

$$
\mathbf{J}=\frac{1}{2 \pi} \nabla P_{3} \times \mathbf{E} .
$$

Formulated in this way, we see that $P_{3}$ should be regarded as an external field.

In fact, any inhomogeneous external field $\phi$ with the same symmetry properties as $P_{3}$, namely, odd under inversion and time reversal, should suffice to generate such a current response. We therefore write $\mathbf{J}=\frac{\chi}{2 \pi} \nabla \phi \times \mathbf{E}$, which is just (2). The response coefficient $\chi$ is the generalization of the magnetoelectric coupling in the insulator, and a more faithful analog of the Hall conductivity - it can now be defined and calculated for metals as well. ${ }^{38}$ For an insulator, $\chi$ will be quantized, yet the precise value of $\chi$ depends on how the field $\phi$ is defined and couples to the system, and therefore by itself will not attain a universal quantized value. This is the one sacrifice we have to make in the alternate formulation of the magnetoelectric response. On the other hand, it will prove a more robust quantity to measure, in a system that may be gapless, and most importantly it will exhibit quantization in an insulator-the key qualitative feature we are after.

Next we will derive (3). The derivation in the body of this paper is not rigorous, and does not apply to gapless systems. It is presented here for the sake of brevity and clarity. In Appendix B we will derive the result with some assumptions, while a general rigorous derivation is left for a future presentation. Using charge conservation $\partial_{t} \rho=-\nabla \cdot \mathbf{J}$, 
as well as Faraday's law $\boldsymbol{\nabla} \times \mathbf{E}=-\partial_{t} \mathbf{B}$, we find from (2)

$$
\rho=-\frac{\chi}{2 \pi} \nabla \phi \cdot \mathbf{B} \text {. }
$$

The second term from (7) has no contribution since $\nabla \cdot \mathbf{B}=0$. Taking $\partial_{c} \phi=h_{c}$, the differential form, (9) becomes

$$
\chi \delta_{a}^{b}=-2 \pi \frac{\partial^{2} \rho}{\partial B^{a} \partial h_{b}}=-2 \pi \frac{\partial^{2} M_{a}}{\partial \mu \partial h_{b}},
$$

where we used the same Maxwell relation as in (5). Note that the magnetoelectric response is found from the magnetization parallel to the direction of the auxiliary field gradient. This should not be surprising as the magnetoelectric effect should not be affected by whether the system is isotropic or not. In an isotropic system, the absence of any other directionality necessitates this outcome. Contracting the $a, b$ indices in $d=$ 3 , we arrive at (3).

A rigorous proof of (3), as well as a generalization to gapless systems, can be derived with some effort. Following standard response-theory techniques, ${ }^{50}$ we can find the nonlinear response analog of the Bastin formula (see Appendix A):

$$
\begin{aligned}
\chi^{\mathrm{I}}= & \frac{4 \pi}{4 !} \epsilon^{a b c} \operatorname{Re}\left\{\operatorname { T r } \left[\delta(H)\left(v_{b} G_{R} v_{\phi}-v_{\phi} G_{R} v_{b}\right)\right.\right. \\
& \left.\left.\times G_{R} v_{c} G_{R} v_{a}\right]\right\}, \\
\chi^{\mathrm{II}}= & 2 \pi \frac{\epsilon^{\mu \nu \lambda \tau}}{4 !} \int_{-\infty}^{+\infty} \frac{d \epsilon}{2 \pi i} f(\epsilon) \\
& \times \operatorname{Tr}\left[v_{\phi} G_{R} v_{\mu} G_{R} v_{\nu} G_{R} v_{\lambda} G_{R} v_{\tau} G_{R}\right]+\text { c.c. },
\end{aligned}
$$

where $v_{a}$ are the velocity operators, $f(\epsilon)$ is the Fermi-Dirac distribution, $\delta(x)$ is the Dirac delta function, and $G_{R}=[\epsilon+$ $i \delta-H]^{-1}$ is the retarded Green's function, which can include any random potential. The chemical potential is included in $H=\cdots-\mu$, so that the Fermi energy is at $\epsilon=0$. The trace is over all degrees of freedom of the system-real (or momentum) space coordinates and internal degrees of freedom. Also, in the expression for the contribution $\chi^{\mathrm{II}}$, all Green's functions depend on the frequency $\epsilon$, while in the expression for $\chi^{\mathrm{I}}$, all Green's functions have $\epsilon=0$. Finally, the velocity $v_{\phi}$ is the conjugate operator to the auxiliary field $H=H_{0}+\int_{\mathbf{x}} \phi(\mathbf{x}) v_{\phi}$. The full details of this derivation we leave for a future presentation. In Appendix B we provide a limited derivation, appropriate for an insulator, with the field $\phi=h_{a} x^{a}$ coupled to a momentum-independent $v_{\phi}$.

\section{IMPLICATIONS FOR EXPERIMENT}

Much as in the case of conductivity, the form (11) distinguishes between Fermi surface contributions $\chi^{\mathrm{I}}$, which vanish for an insulator, and the contribution $\chi^{\mathrm{II}}$, which turns out to be the second derivative of the orbital magnetization, satisfying (3),

$$
\chi^{\mathrm{II}}=-\frac{2 \pi}{3} \frac{\partial^{2} M_{a}}{\partial \mu \partial h_{a}} .
$$

As we already discussed in the Introduction, our result (12), much like the Streda formula (5), indicates that $\chi$ can be obtained by measuring the second derivative of the (orbital) magnetization. As noted earlier, at least in the insulating case, the Zeeman contribution to the magnetization should

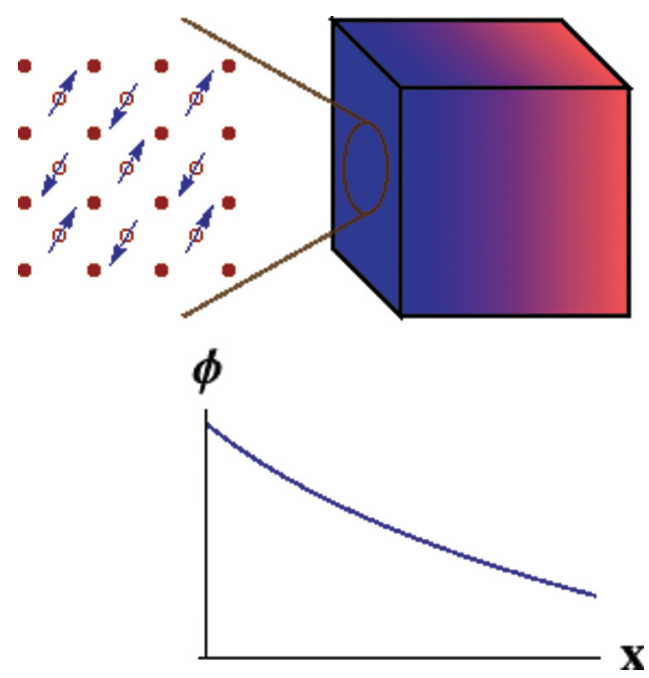

FIG. 1. (Color online) Measuring the topological magnetoelectric response with antiferromagnetic (AFM) order assuming the role of the auxiliary field $\phi$, odd under both inversion and time reversal. At the microscopic level, some of the ions are nonmagnetic (denoted by full circles), while others are magnetic (denoted by open circles), and form AFM order (denoted by the arrows). A slow gradient in $\phi$ in combination with the electromagnetic field generates a magnetoelectric response. The $\phi$ gradient indicated in the graph at the bottom is represented in the image of the material by a change of shade (light red to light blue).

not vary with the chemical potential, and measuring the full magnetization instead of the orbital magnetization alone will yield the same result. It is then conceptually straightforward to measure the magnetization and vary the chemical potential. The auxiliary field gradient $h_{a}$, on the other hand, is at this point an abstract object we defined for our theoretical needs. We turn our attention now to discussing how $\phi$ can be realized. First, given that $\phi$ must be odd under time reversal and inversion, it can appear when antiferromagnetic (AFM) order is present in the material. It is not unimaginable that a topological insulator material could be stuffed with magnetic atoms that realize AFM order in the material. Second, we need $\phi$ to vary (slowly) in space, as illustrated in Fig. 1. This can occur naturally in AFM order, as it tends to form magnetic domains. More difficult will be controlling and varying the strength of the AFM field. This can be done by changing the temperature of the system, and for better control of it, it should be sufficiently close to the critical temperature of the AFM order.

If the topological insulator lacks inversion symmetry to begin with, a field breaking time-reversal symmetry alone should suffice to generate $\phi$. In this case a simple inhomogeneous Zeeman field $B$ could be used, with $\phi \sim \nabla B$. However, a vast majority of the currently known topological insulators are inversion symmetric, and we therefore focus on this case in the current paper.

\section{CALCULATION FOR A MATERIAL EXAMPLE}

We now turn to a concrete example for realizing $\phi$ as a spatially varying Zeeman field in $\mathrm{Bi}_{2} \mathrm{Si}_{3}$. The effective low-energy continuum model derived for $\mathrm{Bi}_{2} \mathrm{Se}_{3}$ in Ref. 51 involves electrons in two orbitals, originating in the $p$ orbitals 


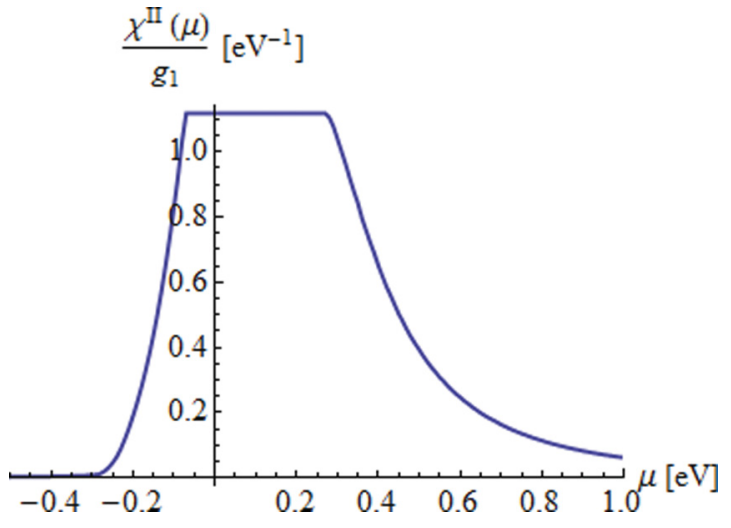

FIG. 2. (Color online) Plot of $\chi^{\mathrm{II}} / g_{1}$ numerically calculated for $\mathrm{Bi}_{2} \mathrm{Se}_{3}$. The $\chi^{\mathrm{II}}$ value is quantized as long as the chemical potential $\mu$ is in the bulk gap. Once $\mu$ is outside the gap, $\chi^{\mathrm{II}}$ is no longer quantized.

of different atoms ( $\mathrm{Bi}$ and $\mathrm{Se}$, respectively). As a consequence, the two orbitals in general will have a different gyromagnetic ratio when coupled to a Zeeman field. Indeed, if the magnetic field $b$ is applied in the direction of the trigonal axis of the $\mathrm{Bi}_{2} \mathrm{Se}_{3}$ crystal (the $z$ direction in the notation of Ref. 51), one finds $H_{\text {Zeeman }}=b \sigma_{3}\left(g_{0}+g_{3} \tau_{3}\right)$, where $\sigma_{1,2,3}$ are the Pauli matrices of the electron spin, and $\tau_{1,2,3}$ are the Pauli matrices describing the orbital degree of freedom. If the magnetic field $b$ varies on the length scale of atomic distances, it will effectively break inversion symmetry in the crystal and allow a more general Zeeman coupling to occur,

$$
H_{\text {Zeeman }}=b_{\mathrm{FM}} \sigma_{3}\left(g_{0}+g_{3} \tau_{3}\right)+b_{\mathrm{AFM}} \sigma_{3}\left(g_{1} \tau_{1}+g_{2} \tau_{2}\right) \text {. }
$$

Here $b_{\mathrm{FM}}$ and $b_{\mathrm{AFM}}$ are "ferromagnetic" (FM) and "antiferromagnetic" (AFM) fields, respectively. The field $b_{\mathrm{AFM}}$ is odd under both time reversal and inversion, and is therefore a suitable realization of $\phi$. Generating it may require antiferromagnetic order, although any magnetic order that varies strongly on a microscopic scale (ferrimagnetism, spin spirals, etc.) will suffice. We also note in passing that we neglect the orbital coupling of the magnetic field we apply here. With a sufficiently weak Zeeman field the flux through a unit cell of the solid will be small, and we can safely neglect it. Using (11) we calculate $\chi^{\mathrm{II}}$ for $\mathrm{Bi}_{2} \mathrm{Se}_{3}$ with all the numerical parameters we need (apart from $g_{1}$ ) taken from Ref. 51. Varying the chemical potential, we find the values plotted in Fig. 2. The most striking feature in plotting $\chi^{\mathrm{II}}$ versus chemical potential is the platea $u$ in its value while $\mu$ is in the gap. Once the chemical potential is outside the gap, the value of $\chi^{\mathrm{II}}$ changes continuously. This will be the most easily discernible experimental signature of the topological insulator-a plateau in the transport coefficient $\chi$-even though its value is not universal.

\section{CONCLUSIONS}

In conclusion, we have found that the magnetoelectric coupling in topological insulators and their gapless counterparts can be related to a third derivative of a thermodynamic potential. Most interestingly, this implies that the topological effects could be measured by probing either charge density or magnetization in equilibrium and in the bulk, rather than from nonequilibrium transport properties of the surface. Our result (3) suggests a conceptually simple way to measure the magnetoelectric response, by measuring the magnetization while varying the chemical potential and the auxiliary field $\phi$, or measuring the charge density while varying the magnetic field and $\phi$. Moreover, our formula holds regardless of whether the system is gapless or gapped, clean or disordered. The measurement we propose, however, is challenging. First and foremost, realizing the auxiliary field is difficult, in the case of $\mathrm{Bi}_{2} \mathrm{Se}_{3}$ requiring the introduction of microscopic AFM order to the bulk of the material, and its careful control. In a topological insulator material lacking inversion symmetry, however, generating the field $\phi$ would be much easier-then an inhomogeneous Zeeman field suffices. As more topological insulator materials continue to be discovered, we are hopeful some of them will be of this variety. Controlling the chemical potential may also be challenging, given that we wish to probe 3D systems. Varying the chemical potential is needed not only to calculate the derivative in (3), but also to detect the most clear-cut evidence for a topological state-the plateau in $\chi^{\mathrm{II}}$, as illustrated in Fig. 2. Finally, the magnetization in our formula is the orbital magnetization, ignoring the Zeeman contributions to the magnetization. In a metal, the Zeeman contribution can vary with the chemical potential, but in an insulator, it will not. Therefore measuring the full magnetization, instead of the orbital magnetization, will yield $\chi^{\text {II }}$ in the insulating state, but in the metal it will yield $\chi^{\text {II }}$ plus some corrections. However, the key qualitative feature is the plateau in $\chi^{\mathrm{II}}$ in the insulating state, which will still show up when measuring the total magnetization instead of the orbital magnetization. It is the quantization of a response coefficient that signifies a topologically incompressible state. Despite these difficulties, our findings allow a different conceptual approach to measuring the magnetoelectric coupling, and it is our sincere hope this insight will be put to use in the laboratory.

\section{ACKNOWLEDGMENTS}

D.L.B. was generously supported by the Sherman Fairchild Foundation. G.R. acknowledges support from DARPA and from the Caltech Institute of Quantum Information and Matter, an NSF Physics Frontiers Center with the support of the Gordon and Betty Moore Foundation.

\section{APPENDIX A: NONLINEAR RESPONSE}

In this Appendix we will briefly describe how one derives the response function to the product of two fields. The external fields $F_{j}(t)$ are coupled to operators $X_{j}$. Coupling an imaginary-time action with three fields $F_{j=1,2,3}$, we have a partition function $Z=\operatorname{Tr}\left[e^{-S-\delta S}\right]$ where

$$
\delta S=-\int_{\tau} \sum_{j=1}^{3} X_{j}(\tau) F_{j}(\tau) .
$$

Here the operators $X_{j}(\tau)$ are in the Heisenberg representation in imaginary time. We assume that the real-time action can be converted to an imaginary-time action with no difficultythat we encounter no poles in the partition function in the continuation of time $t$ to the complex plane. 
The expectation value of the operator $X_{1}$ is given by

$$
\begin{aligned}
\left\langle X_{1}\right\rangle & =\left.\frac{\delta \log (Z)}{\delta F_{1}}\right|_{F_{1}=0} \\
& =\text { equilibrium }+ \text { linear response }+ \text { quadratic response } \\
& =0+O\left(F_{2}+F_{3}\right)+O\left(F_{2} F_{3}\right)+\cdots .
\end{aligned}
$$

The quadratic response coefficient will be given by

$$
\begin{aligned}
u & =\frac{1}{2}\left[\frac{\delta^{2}\left\langle X_{1}\right\rangle}{\delta F_{2} \delta F_{3}}+\frac{\delta^{2}\left\langle X_{1}\right\rangle}{\delta F_{3} \delta F_{2}}\right] \\
& =\left.\frac{1}{2} \frac{\delta^{3} \log (Z)}{\delta F_{1} \delta F_{2} \delta F_{3}}\right|_{F_{j}=0}+\left(F_{2} \leftrightarrow F_{3}\right) \\
& =\left.\frac{1}{2 Z} \frac{\delta^{3} Z}{\delta F_{1} \delta F_{2} \delta F_{3}}\right|_{F_{j}=0}+\left(F_{2} \leftrightarrow F_{3}\right),
\end{aligned}
$$

where the last equality follows from $\left.\frac{\delta Z}{\delta F_{j}}\right|_{F_{j}=0}=0$ - the expectation values for $X_{1,2,3}$ are assumed to vanish in equilibrium. Therefore, we can immediately relate the response coefficient $u$ to a correlation function

$$
\begin{aligned}
u & =\frac{1}{2 Z} \operatorname{Tr}\left[X_{1} X_{2} X_{3} e^{-S}\right]+(2 \leftrightarrow 3) \\
& =\frac{1}{2}\left[\left\langle X_{1} X_{2} X_{3}\right\rangle+\left\langle X_{1} X_{3} X_{2}\right\rangle\right] .
\end{aligned}
$$

If this response function were derived using real time instead of imaginary time, the correlation function would consist of a double commutator,

$$
u \sim\left\langle\left[\left[X_{1}, X_{2}\right], X_{3}\right]\right\rangle .
$$

The imaginary-time formulation is more convenient as it eliminates the commutator.

The correlation function will only depend on the time differences, and we shall therefore Fourier transform from imaginary time to the (bosonic) Mastubara frequency. The correlation function we want to calculate is then

$$
u\left(i \omega, i \omega^{\prime}\right)=\frac{1}{2}\left\langle X_{1}\left(-i \omega-i \omega^{\prime}\right) X_{2}(i \omega) X_{3}\left(i \omega^{\prime}\right)\right\rangle+(2 \leftrightarrow 3),
$$

described by the Feynman diagram in Fig. 3. Using the Green's function $G^{-1}\left(i E_{n}\right)=i E_{n}-H$, we have

$$
\begin{aligned}
u\left(i \omega, i \omega^{\prime}\right)= & T \sum_{n} \operatorname{Tr}\left[X_{1} G\left(i E_{n}\right) X_{2} G\left(i E_{n}+i \omega\right)\right. \\
& \left.\times X_{3} G\left(i E_{n}+i \omega+i \omega^{\prime}\right)\right]+(2 \leftrightarrow 3) \\
= & T \sum_{n} P\left(i E_{n}, i E_{n}+i \omega, i E_{n}+i \omega+i \omega^{\prime}\right),
\end{aligned}
$$

where $E_{n}$ are fermionic Matusbara frequencies, and the trace is over all degrees of freedom in the Hamiltonian. In addition, $X_{j}$ are now in the Schrödinger picture and have no time or frequency dependence.

The response function $u$ in real time must be causal, and therefore we will have to take $i \omega \rightarrow \omega+i \delta$ and $i \omega^{\prime} \rightarrow \omega^{\prime}+$ $i \delta$. The intuitive way to understand this is that the quadratic response must be to two perturbations in the past, and therefore both external fields must be retarded. One should think of the process described in the diagram as two bosons coming in with

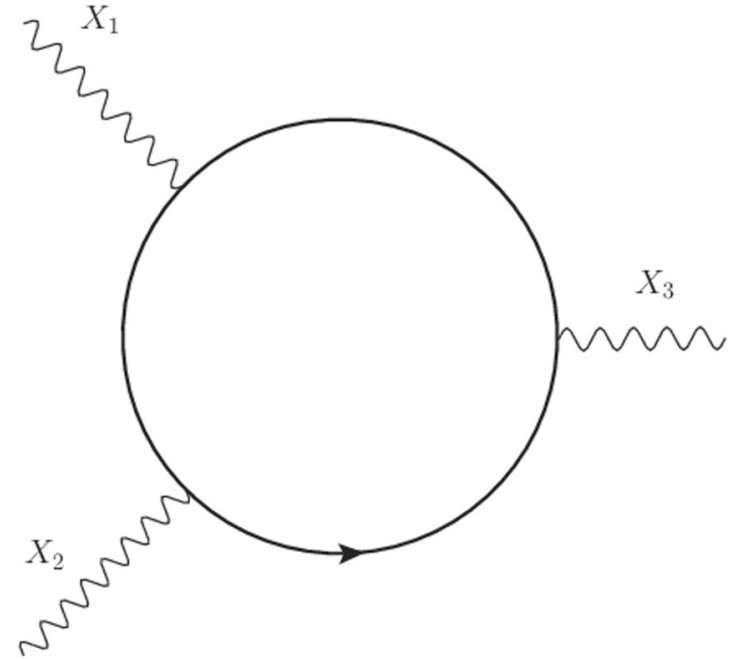

FIG. 3. Nonlinear response from the three-legged bubble. From the particle physics point of view, two bosons are coming in, getting absorbed by a fermion with vertices $X_{2,3}$, and one boson is being emitted by the fermion from vertex $X_{1}$. From the response-theory point of view, two perturbing fields couple to a fermionic system through the operators $X_{2,3}$ and produce an expectation value for the operator $X_{1}$.

frequencies $\omega, \omega^{\prime}$, being absorbed by fermions, and one boson coming out with frequency $\omega+\omega^{\prime}$.

We will now switch from imaginary to real frequencies. The single-particle Green's function

$$
G\left(i E_{n}\right)=\left[i E_{n}-H\right]^{-1}
$$

gets replaced by

$$
G(z)=\{z-H+i \delta \operatorname{sgn}[\operatorname{Im}(z)]\}^{-1},
$$

with the branch cut at $\operatorname{Im}(z)=0$ obviated by the new term involving $0<\delta \rightarrow 0$. It is worth noting at this point that even for a dressed Green's function we will require the same branch cut, since it is the manifestation of causality. The form of the Green's function, with no self-energy, holds strictly only for a noninteracting Hamiltonian, but for our needs this will suffice. Using the Matsubara sum identity

$$
T \sum_{E_{n}} h\left(i E_{n}\right)=\frac{-1}{2 \pi i} \oint d z f(z) h(z),
$$

where $f(z)=\left[1+e^{\beta(z-\mu)}\right]^{-1}$ is the Fermi-Dirac distribution, and assuming that the current vertex does not have any branch cuts, the Matsubara summation has to be broken up into four contours in different domains, divided by the three branch cuts at $\operatorname{Im}(z)=0, \operatorname{Im}(z+i \omega)=0$, and $\operatorname{Im}\left(z+i \omega+i \omega^{\prime}\right)=0$. In each domain all of the poles not in $f(z)$ are outside the domain, since the imaginary part of the denominator in the Green's function never vanishes in each domain. The $X_{j}$ operators do not have any poles, as they are independent of frequency for a noninteracting Hamiltonian. Therefore, the Matsubara sum in the four separate domains can be deformed to contour integrals over the entire extent of each domain (see Fig. 4). Assuming without loss of generality $\omega>0$ and $\omega^{\prime}>0$ (since we will be 
sending them to zero at the end), this yields for us

$$
\begin{aligned}
u\left(i \omega, i \omega^{\prime}\right)= & \sum_{j=1}^{4} \oint_{C_{j}} \frac{i d z}{2 \pi} f(z) P\left(z, z+i \omega, z+i \omega+i \omega^{\prime}\right) \\
= & \int_{-\infty}^{+\infty} \frac{i d \epsilon}{2 \pi}\left\{\left[P\left(\epsilon+i \delta, \epsilon+i \omega, \epsilon+i \omega+i \omega^{\prime}\right)-P\left(\epsilon-i \delta, \epsilon+i \omega, \epsilon+i \omega+i \omega^{\prime}\right)\right] f(\epsilon)+\left[P\left(\epsilon-i \omega, \epsilon+i \delta, \epsilon+i \omega^{\prime}\right)\right.\right. \\
& \left.-P\left(\epsilon-i \omega, \epsilon-i \delta, \epsilon+i \omega^{\prime}\right)\right] f(\epsilon-i \omega)+\left[P\left(\epsilon-i \omega-i \omega^{\prime}, \epsilon-i \omega^{\prime}, \epsilon+i \delta\right)-P\left(\epsilon-i \omega-i \omega^{\prime}, \epsilon-i \omega^{\prime}, \epsilon-i \delta\right)\right] \\
& \left.\times f\left(\epsilon-i \omega-i \omega^{\prime}\right)\right\} .
\end{aligned}
$$

Since $\omega$ and $\omega^{\prime}$ are bosonic Matsubara frequencies, we have $f(\epsilon-i \omega)=f(\epsilon)$ and $f\left(\epsilon-i \omega-i \omega^{\prime}\right)=f(\epsilon)$. This together with the Wick rotations $i \omega \rightarrow \omega+i \delta$ and $i \omega^{\prime} \rightarrow \omega^{\prime}+i \delta$ then yields

$$
\begin{aligned}
u\left(\omega, \omega^{\prime}\right)= & \int_{-\infty}^{+\infty} \frac{i d \epsilon}{2 \pi} f(\epsilon)\left\{\left[P\left(\epsilon+i \delta, \epsilon+\omega+i \delta, \epsilon+\omega+\omega^{\prime}+i \delta\right)-P\left(\epsilon-i \delta, \epsilon+\omega+i \delta, \epsilon+\omega+\omega^{\prime}+i \delta\right)\right]\right. \\
& +\left[P\left(\epsilon-\omega-i \delta, \epsilon+i \delta, \epsilon+\omega^{\prime}+i \delta\right)-P\left(\epsilon-\omega-i \delta, \epsilon-i \delta, \epsilon+\omega^{\prime}+i \delta\right)\right] \\
& \left.+\left[P\left(\epsilon-\omega-\omega^{\prime}-i \delta, \epsilon-\omega^{\prime}-i \delta, \epsilon+i \delta\right)-P\left(\epsilon-\omega-\omega^{\prime}-i \delta, \epsilon-\omega^{\prime}-i \delta, \epsilon-i \delta\right)\right]\right\} .
\end{aligned}
$$

Now we can identify

$$
\begin{aligned}
P\left(\epsilon+i \delta, \epsilon+\omega+i \delta, \epsilon+\omega+\omega^{\prime}+i \delta\right) & =P_{R R R}\left(\epsilon, \epsilon+\omega, \epsilon+\omega+\omega^{\prime}\right) \\
& =\frac{1}{2} \operatorname{Tr}\left[X_{1} G_{R}(\epsilon) X_{2} G_{R}(\epsilon+\omega) X_{3} G_{R}\left(\epsilon+\omega+\omega^{\prime}\right)\right]+(2 \leftrightarrow 3)
\end{aligned}
$$

as involving three retarded Green's functions, and in similar ways identify all the other terms, as products of retarded and advanced Green's functions $G_{R, A}(\epsilon)=[\epsilon \pm i \delta-H]^{-1}$. Shifting the integration variable in the second and third terms

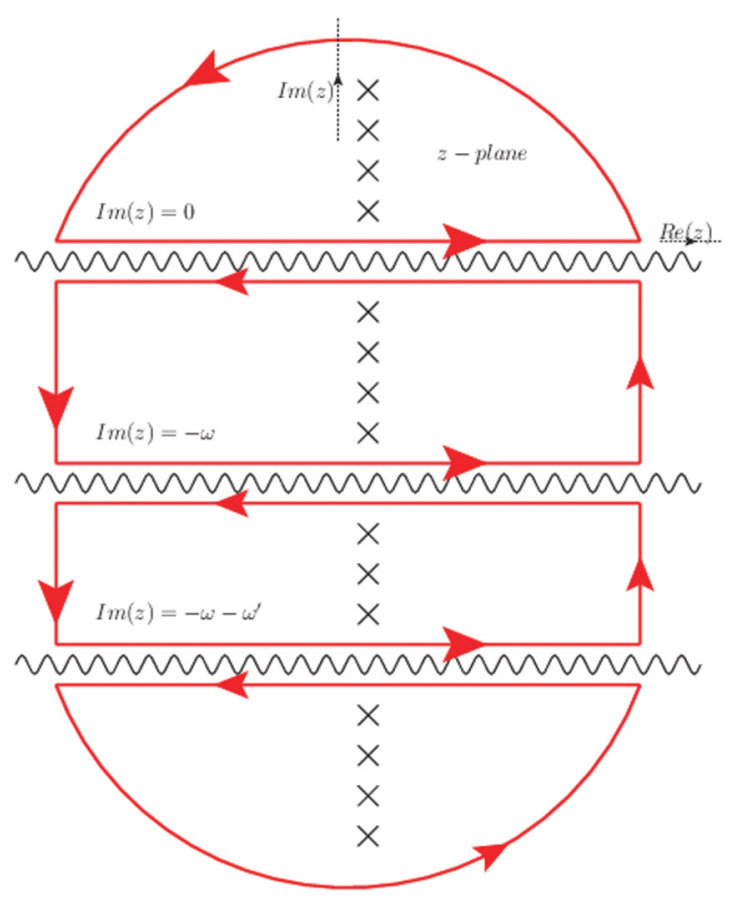

FIG. 4. (Color online) Integration contours in the complex plane for the frequency $z$. Fermionic Matsubara frequencies are the poles marked by (blue) crosses, the three branch cuts are denoted by a wavy (black) line, and the four integration contours are marked by the (red) loops with arrows. we can arrive at

$$
\begin{aligned}
u\left(\omega, \omega^{\prime}\right)= & \int_{-\infty}^{+\infty} \frac{i d \epsilon}{2 \pi}\left[f(\epsilon)\left(P_{R R R}-P_{A R R}\right)\right. \\
& +f(\epsilon+\omega)\left(P_{A R R}-P_{A A R}\right) \\
& \left.+f\left(\epsilon+\omega+\omega^{\prime}\right)\left(P_{A A R}-P_{A A A}\right)\right]
\end{aligned}
$$

where all $P$ functions depend on the trio $(\epsilon, \epsilon+\omega, \epsilon+\omega+$ $\left.\omega^{\prime}\right)$. Grouping together the terms by the number of retarded and advanced Green's functions, we find

$$
\begin{aligned}
u\left(\omega, \omega^{\prime}\right)= & \int_{-\infty}^{+\infty} \frac{i d \epsilon}{2 \pi}\left\{[f(\epsilon+\omega)-f(\epsilon)] P_{A R R}\right. \\
& +\left[f\left(\epsilon+\omega+\omega^{\prime}\right)-f(\epsilon+\omega)\right] P_{A A R} \\
& \left.+\left[f(\epsilon) P_{R R R}-f\left(\epsilon+\omega+\omega^{\prime}\right) P_{A A A}\right]\right\} .
\end{aligned}
$$

This is the most general form of nonlinear response to two independent fields.

\section{APPENDIX B: DERIVATION OF THE THERMODYNAMIC RELATION}

In the previous section, we analyzed the general expression (A15) for a quadratic response to two fields (in our case $F_{a b}$ and $h_{c}$ ), captured by the Feynman diagram of Fig. 3. In this section we will use (A15) to prove the relation (12).

We will be interested only in the low-frequency limit, and in fact we will keep terms only to linear order in the frequencies $\omega, \omega^{\prime}$. We first shift the integration variable in the last term in (A15), $\epsilon \rightarrow \epsilon-\omega$, to get

$$
\begin{aligned}
& {\left[f(\epsilon) P_{R R R}-f\left(\epsilon+\omega+\omega^{\prime}\right) P_{A A A}\right]} \\
& \quad \rightarrow f(\epsilon-\omega) P_{R R R}\left(\epsilon-\omega, \epsilon, \epsilon+\omega^{\prime}\right) \\
& \quad-f\left(\epsilon+\omega^{\prime}\right) P_{A A A}\left(\epsilon-\omega, \epsilon, \epsilon+\omega^{\prime}\right),
\end{aligned}
$$


and then expanding in $\omega, \omega^{\prime}$ we reach

$$
\begin{aligned}
u\left(\omega, \omega^{\prime}\right) \approx & \int_{-\infty}^{+\infty} \frac{i d \epsilon}{2 \pi}\left(f^{\prime}(\epsilon)\left\{\omega\left[P_{A R R}(\epsilon, \epsilon, \epsilon)-P_{R R R}(\epsilon, \epsilon, \epsilon)\right]+\omega^{\prime}\left[P_{A A R}(\epsilon, \epsilon, \epsilon)-P_{A A A}(\epsilon, \epsilon, \epsilon)\right]\right\}\right. \\
& \left.+f(\epsilon)\left[P_{R R R}\left(\epsilon-\omega, \epsilon, \epsilon+\omega^{\prime}\right)-P_{A A A}\left(\epsilon-\omega, \epsilon, \epsilon+\omega^{\prime}\right)\right]\right) \\
\approx & \int_{-\infty}^{+\infty} \frac{i d \epsilon}{2 \pi}\left\{f^{\prime}(\epsilon)\left[\omega\left(P_{A R R}-P_{R R R}\right)+\omega^{\prime}\left(P_{A A R}-P_{A A A}\right)\right]+f(\epsilon)\left[\frac{\omega^{\prime}}{2} \operatorname{Tr}\left[X_{1} G_{R}\left(X_{2} G_{R} X_{3}+X_{3} G_{R} X_{2}\right) \partial_{\epsilon} G_{R}\right]\right.\right. \\
& \left.\left.-\frac{\omega}{2} \operatorname{Tr}\left[X_{1} \partial_{\epsilon} G_{R}\left(X_{2} G_{R} X_{3}+X_{3} G_{R} X_{2}\right) G_{R}\right]+P_{R R R}-(R \rightarrow A)\right]\right\}
\end{aligned}
$$

where in the final form all Green's functions depend on the energy $\epsilon, G_{R, A}=G_{R, A}(\epsilon)$ and $P_{R R R}=P_{R R R}(\epsilon, \epsilon, \epsilon)$. Now we split this expression into terms involving a derivative of the Fermi-Dirac function $u^{\mathrm{I}}$, and all the rest $u^{\mathrm{II}}=u-u^{\mathrm{I}}$. The two contributions are

$$
\begin{aligned}
u^{\mathrm{I}}\left(\omega, \omega^{\prime}\right)= & \omega \int_{-\infty}^{+\infty} \frac{i d \epsilon}{2 \pi} f^{\prime}(\epsilon)\left[P_{A R R}(\epsilon, \epsilon, \epsilon)-P_{R R R}(\epsilon, \epsilon, \epsilon)\right]+\omega^{\prime} \int_{-\infty}^{+\infty} \frac{i d \epsilon}{2 \pi} f^{\prime}(\epsilon)\left[P_{A A R}(\epsilon, \epsilon, \epsilon)-P_{A A A}(\epsilon, \epsilon, \epsilon)\right] \\
u^{\mathrm{II}}\left(\omega, \omega^{\prime}\right)= & \int_{-\infty}^{+\infty} \frac{i d \epsilon}{2 \pi} f(\epsilon)\left[\frac{\omega^{\prime}}{2} \operatorname{Tr}\left[X_{1} G_{R}\left(X_{2} G_{R} X_{3}+X_{3} G_{R} X_{2}\right)\left(\partial_{\epsilon} G_{R}\right)\right]-\frac{\omega}{2} \operatorname{Tr}\left[X_{1}\left(\partial_{\epsilon} G_{R}\right)\left(X_{2} G_{R} X_{3}+X_{3} G_{R} X_{2}\right) G_{R}\right]\right. \\
& \left.+P_{R R R}-(R \rightarrow A)\right] .
\end{aligned}
$$

At zero temperature $f^{\prime}(\epsilon)=-\delta(\epsilon)$, leading to

$$
\begin{aligned}
\left.u^{\mathrm{I}}\right|_{T=0}= & \frac{\omega}{2 \pi i}\left[P_{A R R}(0,0,0)-P_{R R R}(0,0,0)\right] \\
& +\frac{\omega^{\prime}}{2 \pi i}\left[P_{A A R}(0,0,0)-P_{A A A}(0,0,0)\right] .
\end{aligned}
$$

Using the identity

$$
G_{A}(\epsilon)-G_{R}(\epsilon)=\frac{1}{2 \pi i} \delta(H-\epsilon),
$$

we can write

$$
\begin{aligned}
\left.u^{\mathrm{I}}\right|_{T=0}= & \frac{\omega}{2}\left[X_{1} \delta(H) X_{2} G_{R}(0) X_{3} G_{R}(0)\right] \\
& -\frac{\omega^{\prime}}{2}\left[X_{1} G_{A}(0) X_{2} G_{A}(0) X_{3} \delta(H)\right]+(2 \leftrightarrow 3),
\end{aligned}
$$

and we see that this term involves contributions only from the Fermi surface. For an insulator, there is zero density of states at the Fermi energy $\epsilon=0$, and this contribution will vanish. More generally, at low temperature and frequency $u^{\mathrm{I}}$ depends only on states close to the Fermi energy.

Now we specialize the calculation to our problem. Consider a band structure perturbed by a random scalar potential,

$$
H=H_{0}(\mathbf{p})+V(\mathbf{x}),
$$

and couple to an auxiliary field with a gradient $\phi=x_{c} h_{c}$ as well as to an electric field, using the gauge $A_{b}=\frac{1}{i \omega} e^{-i \omega t} E_{b}$,

$$
H_{1}=x_{c} v_{\phi} h^{c}+\frac{1}{i \omega} e^{-i \omega t} E^{b} v_{b} .
$$

We will calculate the current response

$$
J_{a}=u \epsilon_{a b c} E^{b} h^{c} .
$$

Comparing this with (2), we identify $u=\frac{\chi}{2 \pi}$. Note that $v_{b}=$ $\frac{\partial H_{0}}{\partial p^{b}}$, and that we assume $v_{\phi}$ is independent of momentum and thus commutes with $x_{a}$. We will have to antisymmetrize in the indices $a, b, c$, and take $\omega \rightarrow 0$ for the dc limit. As mentioned above, in the insulator case we have $u^{\mathrm{I}}=0$, and we need only calculate $u^{\mathrm{II}}$.

We identify $X_{1}=v_{a}, X_{2}=\frac{1}{i \omega} v_{b}$, and $X_{3}=x_{c} v_{\phi}$. The $P_{R R R}$ term will vanish since is symmetric under $a \leftrightarrow b$,

$$
\begin{aligned}
\epsilon^{a b c} & P_{R R R} \\
& \rightarrow \frac{\epsilon^{a b c}}{2 i \omega} \operatorname{Tr}\left[v_{a} G_{R} v_{b} G_{R} x_{c} v_{\phi} G_{R}+v_{a} G_{R} x_{c} v_{\phi} G_{R} v_{b} G_{R}\right] \\
\quad & =\frac{\epsilon^{a b c}}{i \omega} \operatorname{Tr}\left[\left(v_{a} G_{R} v_{b}+v_{b} G_{R} v_{a}\right) G_{R} x_{c} v_{\phi} G_{R}\right]
\end{aligned}
$$

We are then left with

$$
\begin{aligned}
u^{\mathrm{II}}= & \frac{\epsilon^{a b c}}{3 !} \int_{-\infty}^{+\infty} \frac{i d \epsilon}{2 \pi} f(\epsilon)\left[\frac{\omega}{2} \operatorname{Tr}\left[v_{a} G_{R} x_{c} v_{\phi} G_{R} \frac{v_{b}}{i \omega}\left(\partial_{\epsilon} G_{R}\right)\right]\right. \\
& \left.-\frac{\omega}{2} \operatorname{Tr}\left(v_{a}\left(\partial_{\epsilon} G_{R}\right) \frac{v_{b}}{i \omega} G_{R} x_{c} v_{\phi} G_{R}\right)-(R \rightarrow A)\right] .
\end{aligned}
$$

Swapping $a \leftrightarrow b$ in the second term, we get

$$
\begin{aligned}
u^{\mathrm{II}}= & \frac{\epsilon^{a b c}}{3 !} \int_{-\infty}^{+\infty} \frac{d \epsilon}{2 \pi} f(\epsilon) \\
& \times \operatorname{Tr}\left[v_{a} G_{R} x_{c} v_{\phi} G_{R} v_{b}\left(\partial_{\epsilon} G_{R}\right)\right]-(R \rightarrow A) .
\end{aligned}
$$

We shall now integrate by parts, to get

$$
\begin{aligned}
u^{\mathrm{II}}= & -\frac{\epsilon^{a b c}}{3 !} \int_{-\infty}^{+\infty} \frac{d \epsilon}{2 \pi} f(\epsilon) \\
& \times \operatorname{Tr}\left\{v_{a}\left[\left(\partial_{\epsilon} G_{R}\right) x_{c} v_{\phi} G_{R}+G_{R} x_{c} v_{\phi}\left(\partial_{\epsilon} G_{R}\right)\right] v_{b} G_{R}\right\} \\
& -\frac{\epsilon^{a b c}}{3 !} \int_{-\infty}^{+\infty} \frac{d \epsilon}{2 \pi} f^{\prime}(\epsilon) \operatorname{Tr}\left[v_{a} G_{R} x_{c} v_{\phi} G_{R} v_{b} G_{R}\right] \\
& -(R \rightarrow A) .
\end{aligned}
$$


This can be reorganized into

$$
\begin{aligned}
u^{\mathrm{II}}= & \frac{\epsilon^{a b c}}{3 !} \int_{-\infty}^{+\infty} \frac{d \epsilon}{2 \pi} f(\epsilon) \operatorname{Tr}\left[G_{R} x_{c} v_{\phi} G_{R} v_{b} G_{R} v_{a} G_{R}\right. \\
& \left.+G_{R} v_{b} G_{R} v_{a} G_{R} x_{c} v_{\phi} G_{R}\right] \\
& -\frac{\epsilon^{a b c}}{3 !} \int_{-\infty}^{+\infty} \frac{d \epsilon}{2 \pi} f^{\prime}(\epsilon) \operatorname{Tr}\left[x_{c} v_{\phi} G_{R} v_{b} G_{R} v_{a} G_{R}\right] \\
& -(R \rightarrow A) .
\end{aligned}
$$

Using the relation $v_{a}=-i\left[x_{a}, H\right]=+i\left[x_{a}, G^{-1}\right]$, for either of $G=G_{R, A}$, we find

$$
G v_{a} G v_{b} G-(a \leftrightarrow b)=i G x_{a} v_{b} G+x_{a} G x_{b}-(a \leftrightarrow b) .
$$

Using this identity, as well as $x_{a} x_{b}-(a \leftrightarrow b)$ and the fact that $v_{\phi}$ commutes with $x_{a}$, we can show that

$$
\begin{aligned}
u^{\mathrm{II}}= & -\frac{\epsilon^{a b c}}{3 !} \int_{-\infty}^{+\infty} \frac{i d \epsilon}{2 \pi} f(\epsilon) \\
& \times \operatorname{Tr}\left[G_{R} x_{c} v_{\phi} G_{R} x_{a} v_{b} G_{R}+G_{R} x_{a} v_{b} G_{R} x_{c} v_{\phi} G_{R}\right] \\
& +\frac{\epsilon^{a b c}}{3 !} \int_{-\infty}^{+\infty} \frac{i d \epsilon}{2 \pi} f^{\prime}(\epsilon) \operatorname{Tr}\left[x_{c} v_{\phi} G_{R} x_{a} v_{b} G_{R}\right] \\
& -(R \rightarrow A)
\end{aligned}
$$

where we have also swapped $a$ and $b$. form

With the further observation that with a Hamiltonian of the

$$
H=H_{0}(\mathbf{p})+V(\mathbf{x})+v_{a} A^{a}+v_{\phi} x_{c} h^{c},
$$

where the vector potential is $A^{a}=-\frac{1}{2} \epsilon^{a b c} x_{b} B_{c}$ and the auxiliary field is $\phi=x_{c} h^{c}$, we can show that

$$
\begin{aligned}
\frac{\partial H}{\partial B_{c}} & =-\frac{\partial G^{-1}}{\partial B_{c}}=-\frac{1}{2} \epsilon^{a b c} v_{a} x_{b}=+\frac{1}{2} \epsilon^{a b c} x_{a} v_{b}, \\
\frac{\partial H}{\partial h^{c}} & =-\frac{\partial G^{-1}}{\partial h^{c}}=v_{\phi} x_{c}=x_{c} v_{\phi} .
\end{aligned}
$$

After some more algebra, using $\partial G=-G \partial G^{-1} G$, we can show that

$$
\frac{\partial^{2} G}{\partial B_{d} \partial h^{c}}=G \frac{\partial H}{\partial B_{d}} G \frac{\partial H}{\partial h^{c}} G+G \frac{\partial H}{\partial h^{c}} G \frac{\partial H}{\partial B_{d}} G,
$$

from which we can find

$$
\begin{aligned}
\operatorname{Tr} & {\left[\frac{\partial^{2} G}{\partial B_{c} \partial h^{c}}\right] } \\
& =\frac{1}{2} \epsilon^{a b c} \operatorname{Tr}\left[G x_{a} v_{b} G x_{c} v_{\phi} G+G x_{c} v_{\phi} G x_{a} v_{b} G\right] .
\end{aligned}
$$

Using this, we arrive at

$$
\begin{aligned}
u^{\mathrm{II}}= & \frac{2}{3 !} \int_{-\infty}^{+\infty} \frac{d \epsilon}{2 \pi i} f(\epsilon) \operatorname{Tr}\left[\frac{\partial^{2}\left(G_{R}-G_{A}\right)}{\partial B_{c} \partial h^{c}}\right] \\
& -\frac{2}{3 !} \int_{-\infty}^{+\infty} \frac{d \epsilon}{2 \pi i} f^{\prime}(\epsilon) \operatorname{Tr}\left[x_{c} v_{\phi} \frac{\partial\left(G_{R}-G_{A}\right)}{\partial B_{c}}\right] .
\end{aligned}
$$

Using $G_{A}-G_{R}=2 \pi i \delta(\epsilon-H)$, we arrive at

$$
\begin{aligned}
u^{\mathrm{II}}= & -\frac{1}{3} \frac{\partial^{2}}{\partial B_{c} \partial h^{c}} \int_{-\infty}^{+\infty} d \epsilon f(\epsilon) \operatorname{Tr}[\delta(\epsilon-H)] \\
& +\frac{1}{3} \frac{\partial}{\partial B_{c}} \int_{-\infty}^{+\infty} d \epsilon f^{\prime}(\epsilon) \operatorname{Tr}\left[x_{c} v_{\phi} \delta(\epsilon-H)\right] .
\end{aligned}
$$

The last term at $T=0$ becomes

$$
-\frac{1}{3} \frac{\partial}{\partial B_{c}} \operatorname{Tr}\left[x_{c} v_{\phi} \delta(H)\right]
$$

and vanishes for an insulator. We are then left with

$$
u^{\mathrm{II}}=-\frac{1}{3} \frac{\partial^{2} \rho}{\partial B_{c} \partial h^{c}},
$$

which gives us

$$
\chi=2 \pi u^{\mathrm{II}}=-\frac{2 \pi}{3} \frac{\partial^{2} \rho}{\partial B_{c} \partial h^{c}},
$$

proving our main result (12).

\section{APPENDIX C: CALCULATING THE MAGNETOELECTRIC RESPONSE COEFFICIENT FOR $\mathrm{Bi}_{2} \mathrm{Se}_{3}$}

In this Appendix we present the details of the calculation of $\chi^{\mathrm{II}}$ for $\mathrm{Bi}_{2} \mathrm{Se}_{3}$. The effective low-energy continuum model for $\mathrm{Bi}_{2} \mathrm{Se}_{3}$ is ${ }^{51}$

$H_{0}=C(\mathbf{q})+M(\mathbf{q}) \gamma_{5}+B_{0} q_{3} \gamma_{4}+A_{0}\left(q_{2} \gamma_{1}-q_{1} \gamma_{2}\right)+o\left(q^{3}\right)$,

where $C(\mathbf{q})=C_{0}-\mu+C_{1} q_{3}^{2}+C_{2}\left(q_{1}^{2}+q_{2}^{2}\right)$, and $M(\mathbf{q})=$ $M_{0}+M_{1} q_{3}^{2}+M_{2}\left(q_{1}^{2}+q_{2}^{2}\right)$. The $\gamma$ matrices are taken as $\gamma_{a=1,2,3}=\sigma_{a} \tau_{1}, \gamma_{4}=\tau_{2}$, and $\gamma_{5}=\tau_{3}$. It is noteworthy that the model $(\mathrm{C} 1)$ with different values for the parameters can be used to describe the model proposed in Ref. 6 for a 3D STI. The authors of Ref. 51 have calculated $g_{0} \approx$ $-21.3 \mu_{B} / 4 \approx-3.1 \times 10^{-4} \mathrm{~T}^{-1} \mathrm{eV}$ and $g_{3} \approx-29.5 \mu_{B} / 4 \approx$ $4.3 \times 10^{-4} \mathrm{~T}^{-1} \mathrm{eV}$, and the remaining parameters in $(\mathrm{C} 1)$ take on the values $A_{0}=3.33 \mathrm{eV} \AA, B_{0}=2.26 \mathrm{eV} \AA, C_{0}=$ $-0.0083 \mathrm{eV}, C_{1}=5.74 \mathrm{eV} \AA^{2}, C_{2}=30.4 \mathrm{eV} \AA^{2}, M_{0}=$ $-0.28 \mathrm{eV}, M_{1}=6.86 \mathrm{eV} \AA^{2}$, and $M_{2}=44.5 \mathrm{eV} \AA^{2}$.

Taking the model Hamiltonian (C1) coupled to the Zeeman fields in (13), we have $H(b)=H_{0}+b \sigma_{3}\left(\sum_{j=0}^{3} g_{j} \tau_{j}\right)$. Using (11), at temperature $T=0$ with $v_{\phi}=\frac{\partial H(b)}{\partial b}$, we find

$$
\begin{aligned}
\chi^{\mathrm{II}}(b & =0) \\
= & -g_{1} 4 i A_{0}^{2} B_{0} \int_{0}^{\infty} \frac{2 \pi q d q}{(2 \pi)^{2}} \int_{-\infty}^{0} d \epsilon \int_{-\infty}^{+\infty} \frac{d z}{2 \pi} \\
& \times \frac{\left[M_{0}-M_{2} q^{2}-M_{1} z^{2}\right]}{\left\{M(q, z)^{2}+A_{0}^{2} q^{2}+B_{0}^{2} z^{2}-[\epsilon+i \delta-C(q, z)]^{2}\right\}^{3}} \\
& + \text { c.c. },
\end{aligned}
$$

where we denote $q^{2}=q_{1}^{2}+q_{2}^{2}$ and $z=q_{3}$. Note here that $\chi$ depends only on $g_{1}$. It is only a very special coupling that produces the completely antisymmetric magnetoelectric response, and this observation tells us that even if we have both $b_{\mathrm{FM}}$ and $b_{\mathrm{AFM}}, \chi$ can be measured since it is a response 
only to $b_{\mathrm{AFM}}$. We perform the energy integral first:

$$
\begin{aligned}
\lim _{\delta \rightarrow 0} \operatorname{Im}\left[\int_{-\infty}^{0} \frac{d \epsilon}{\left(E^{2}-(\epsilon-C+i \delta)^{2}\right)^{3}}\right] \\
=\frac{3 \pi}{16 E^{5}}\left\{\frac{2}{\pi} \operatorname{Im}\left[\tanh ^{-1}\left(\frac{-C}{E}\right)\right]-1\right\} \\
=-\frac{3 \pi \Theta\left(E^{2}-C^{2}\right)}{16 E^{5}}
\end{aligned}
$$

where $E^{2}=M(q, z)^{2}+A_{0}^{2} q^{2}+B_{0}^{2} z^{2}$, and we have used

$$
\frac{2}{\pi} \operatorname{Im}\left[\tanh ^{-1}(x)\right]=\left\{\begin{array}{ll}
1, & |x|>1, \\
0, & |x|<1,
\end{array}=\Theta\left(x^{2}-1\right) .\right.
$$

We are now left with

$$
\begin{aligned}
\chi^{\mathrm{II}}(b=0)= & -g_{1} \frac{3 \pi}{2} A_{0}^{2} B_{0} \int_{0}^{\infty} \frac{2 \pi q d q}{(2 \pi)^{2}} \int_{-\infty}^{+\infty} \frac{d z}{2 \pi} \\
& \times \Theta\left(E^{2}-C(q, z)^{2}\right) \frac{\left[M_{0}-M_{2} q^{2}-M_{1} z^{2}\right]}{E^{5}} .
\end{aligned}
$$

Note that the combination $\chi^{\mathrm{II}} \phi=\chi^{\mathrm{II}} b_{\mathrm{AFM}}$ is dimensionless, as we required. By varying the chemical potential, we produce Fig. 2.

\section{APPENDIX D: METHOD OF MEASURING ZEEMAN COUPLING TO AFM ORDER}

The Zeeman coupling to the AFM field $g_{1}$ used in (13) is unknown. While it should be possible to calculate it, a better approach may be to measure it experimentally, in the following manner. Starting from zero, increase the auxiliary field gradient in the bulk, until zero modes appear in the bulk. This will have a distinct spectroscopic signature. Take our model for $\mathrm{Bi}_{2} \mathrm{Se}_{3}$, (C1), with $C_{0} \approx 0$ and keep only terms to linear order in $q$,

$$
\begin{aligned}
H= & M_{0} \gamma_{5}+B_{0} q_{3} \gamma_{4}+A_{0}\left(q_{2} \gamma_{1}-q_{1} \gamma_{2}\right)+g_{1} b_{\mathrm{AFM}}(\mathbf{x}) \gamma_{3} \\
& +o\left(q^{2}\right),
\end{aligned}
$$

where we have used $\gamma_{3}=\sigma_{3} \tau_{1}$. Squaring the Hamiltonian, and assuming that $b_{\mathrm{AFM}}$ varies only in the $x$ direction, we find

$$
\begin{aligned}
H^{2}= & M_{0}^{2}+A_{0}\left(q_{1}^{2}+q_{2}^{2}\right)+B_{0}^{2} q_{3}^{2}+\left(g_{1} b_{\mathrm{AFM}}\right)^{2} \\
& +i A_{0} g_{1} \gamma_{2} \gamma_{3} \partial_{x} b_{\mathrm{AFM}}(x) .
\end{aligned}
$$

From this we can find

$$
\begin{aligned}
& \left\{H^{2}-\left[M_{0}^{2}+A_{0}\left(q_{1}^{2}+q_{2}^{2}\right)+B_{0}^{2} q_{3}^{2}+\left(g_{1} b_{\mathrm{AFM}}\right)^{2}\right]\right\}^{2} \\
& \quad=A_{0}^{2} g_{1}^{2}\left[\partial_{x} b_{\mathrm{AFM}}(x)\right]^{2} .
\end{aligned}
$$

The term on the right-hand side is $o\left(b_{\mathrm{AFM}}^{2}\right)$, while the last term on the left-hand side is $o\left(b_{\mathrm{AFM}}^{4}\right)$, and we neglect it. If we now look for $E=0$ solutions we find these are possible only when

$$
\left|\partial_{x} b_{\mathrm{AFM}}(x)\right| \approx \frac{M_{0}}{A_{0} g_{1}}=\frac{1}{\xi g_{1}} .
$$

We identify $\xi=\frac{A_{0}}{M_{0}}$ as the skin depth of the Jackiw-Rebbi surface state solutions, ${ }^{52}$ and in this way we see that as the gradient is increased, when it hits the value $g_{1}\left|\nabla b_{\mathrm{AFM}}(x)\right| \approx \xi^{-1}$, gapless states appear tied to this gradient. This would suggest that as we increase the gradient we could observe spectral flow, and this could perhaps be measured by absorption experiments.
${ }^{1}$ K. v. Klitzing, G. Dorda, and M. Pepper, Phys. Rev. Lett. 45, 494 (1980).

${ }^{2}$ M. B. Hastings and S. Michalakis, arXiv:0911.4706.

${ }^{3}$ C. L. Kane and E. J. Mele, Phys. Rev. Lett. 95, 226801 (2005).

${ }^{4}$ C. L. Kane and E. J. Mele, Phys. Rev. Lett. 95, 146802 (2005).

${ }^{5}$ B. A. Bernevig, T. L. Hughes, and S.-C. Zhang, Science 314, 1757 (2006).

${ }^{6}$ L. Fu, C. L. Kane, and E. J. Mele, Phys. Rev. Lett. 98, 106803 (2007).

${ }^{7}$ J. E. Moore and L. Balents, Phys. Rev. B 75, 121306 (2007).

${ }^{8}$ L. Smrcka and P. Streda, J. Phys. C 10, 2153 (1977).

${ }^{9}$ P. Streda, J. Phys. C 15, L717 (1982).

${ }^{10}$ R. Shindou and S. Murakami, Phys. Rev. B 79, 045321 (2009).

${ }^{11}$ X. -L. Qi, T. L. Hughes, and S.-C. Zhang, Phys. Rev. B 78, 195424 (2008).

${ }^{12}$ A. M. Essin, J. E. Moore, and D. Vanderbilt, Phys. Rev. Lett. 102, 146805 (2009).

${ }^{13}$ D. Hsieh, D. Qian, L. Wray, Y. Xia, Y. S. Hor, R. J. Cava, and M. Z. Hasan, Nature (London) 452, 970 (2008).

${ }^{14}$ Y. Xia, D. Qian, D. Hsieh, L. Wray, A. Pal, H. Lin, A. Bansil, D. Grauer, Y. S. Hor, R. J. Cava et al., Nat. Phys. 5, 398 (2009).

${ }^{15}$ D. Hsieh, Y. Xia, D. Qian, L. Wray, F. Meier, J. H. Dil, J. Osterwalder, L. Patthey, A. V. Fedorov, H. Lin et al., Phys. Rev. Lett. 103, 146401 (2009).
${ }^{16}$ D. Hsieh, Y. Xia, L. Wray, D. Qian, A. Pal, J. H. Dil, J. Osterwalder, F. Meier, G. Bihlmayer, C. L. Kane et al., Science 323, 919 (2009).

${ }^{17}$ D. Hsieh, L. Wray, D. Qian, Y. Xia, J. H. Dil, F. Meier, L. Patthey, J. Osterwalder, G. Bihlmayer, Y. S. Hor et al., New J. Phys. 12, 125001 (2010).

${ }^{18}$ J. G. Analytis, J.-H. Chu, Y. Chen, F. Corredor, R. D. McDonald, Z. X. Shen, and I. R. Fisher, Phys. Rev. B 81, 205407 (2010).

${ }^{19}$ K. Eto, Z. Ren, A. A. Taskin, K. Segawa, and Y. Ando, Phys. Rev. B 81, 195309 (2010).

${ }^{20}$ J. J. Hamlin, J. R. Jeffries, N. P. Butch, P. Syers, D. A. Zocco, S. T. Weir, Y. K. Vohra, J. Paglione, and M. B. Maple, J. Phys.: Condens. Matter 24, 035602 (2012)

${ }^{21}$ D. Kim, S. Cho, N. P. Butch, P. Syers, K. Kirshenbaum, S. Adam, J. Paglione, and M. S. Fuhrer, Nat. Phys. 8, 460 (2012).

${ }^{22}$ S. Cho, N. P. Butch, J. Paglione, and M. S. Fuhrer, Nano Lett. 11, 1925 (2011).

${ }^{23}$ H. Steinberg, J.-B. Laloë, V. Fatemi, J. S. Moodera, and P. JarilloHerrero, Phys. Rev. B 84, 233101 (2011).

${ }^{24}$ H. Steinberg, D. R. Gardner, Y. S. Lee, and P. Jarillo-Herrero, Nano Lett. 10, 5032 (2010).

${ }^{25}$ X.-L. Qi and S.-C. Zhang, Rev. Mod. Phys. 83, 1057 (2011).

${ }^{26}$ W.-K. Tse and A. H. MacDonald, Phys. Rev. Lett. 105, 057401 (2010).

${ }^{27}$ Y. Lan, S. Wan, and S.-C. Zhang, Phys. Rev. B 83, 205109 (2011). 
${ }^{28}$ A. Karch, Phys. Rev. Lett. 103, 171601 (2009).

${ }^{29}$ A. D. LaForge, A. Frenzel, B. C. Pursley, T. Lin, X. Liu, J. Shi, and D. N. Basov, Phys. Rev. B 81, 125120 (2010).

${ }^{30}$ J. Maciejko, X.-L. Qi, H. D. Drew, and S.-C. Zhang, Phys. Rev. Lett. 105, 166803 (2010).

${ }^{31}$ G. Tkachov and E. M. Hankiewicz, Phys. Rev. B 84, 035405 (2011).

${ }^{32}$ I. Garate and M. Franz, Phys. Rev. Lett. 104, 146802 (2010).

${ }^{33}$ A. B. Sushkov, G. S. Jenkins, D. C. Schmadel, N. P. Butch, J. Paglione, and H. D. Drew, Phys. Rev. B 82, 125110 (2010).

${ }^{34}$ J. Zang and N. Nagaosa, Phys. Rev. B 81, 245125 (2010).

${ }^{35}$ G. S. Jenkins, A. B. Sushkov, D. C. Schmadel, N. P. Butch, P. Syers, J. Paglione, and H. D. Drew, Phys. Rev. B 82, 125120 (2010).

${ }^{36}$ E. Witten, Phys. Lett. B 86, 283 (1979).

${ }^{37}$ G. Rosenberg and M. Franz, Phys. Rev. B 82, 035105 (2010).

${ }^{38}$ D. L. Bergman, Phys. Rev. Lett. 107, 176801 (2011).

${ }^{39}$ M. Barkeshli and X.-L. Qi, Phys. Rev. Lett. 107, 206602 (2011).

${ }^{40}$ R. Karplus and J. M. Luttinger, Phys. Rev. 95, 1154 (1954).

${ }^{41}$ T. Jungwirth, Q. Niu, and A. H. MacDonald, Phys. Rev. Lett. 88, 207208 (2002).
${ }^{42}$ M. Onoda and N. Nagaosa, J. Phys. Soc. Jpn. 71, 19 (2002).

${ }^{43}$ N. Nagaosa, J. Sinova, S. Onoda, A. H. MacDonald, and N. P. Ong, Rev. Mod. Phys. 82, 1539 (2010).

${ }^{44}$ A. Bastin, C. Lewiner, O. Betbeder-matibet, and P. Nozieres, J. Phys. Chem. Solids 32, 1811 (1971).

${ }^{45}$ A. Crépieux and P. Bruno, Phys. Rev. B 64, 014416 (2001).

${ }^{46}$ A. Widom, Phys. Lett. A 90, 474 (1982).

${ }^{47}$ P. Streda and L. Smrcka, J. Phys. C 16, L895 (1983).

${ }^{48}$ A. Usher and M. Elliott, J. Phys.: Condens. Matter 21, 103202 (2009).

${ }^{49}$ S. Wiegers, M. Specht, E. Bibow, L. Lvy, S. Melinte, E. Grivei, V. Bayot, M. Simmons, D. Ritchie, M. Shayegan et al., Physica B 256-258, 16 (1998).

${ }^{50}$ G. D. Mahan, Many-Particle Physics (Plenum Press, New York, 1981).

${ }^{51}$ C.-X. Liu, X.-L. Qi, H. J. Zhang, X. Dai, Z. Fang, and S.-C. Zhang, Phys. Rev. B 82, 045122 (2010).

${ }^{52}$ R. Jackiw and C. Rebbi, Phys. Rev. D 13, 3398 (1976). 